\title{
Arbeitsorganisation und Führungskultur
}

Margit Christiansen

9.1 Einleitung -124

9.2 Situation in der stationären Langzeitpflege - 125

9.2.1 Strukturelle Bedingungen - 125

9.2.2 Präferenzen der Pflegekräfte - 126

9.3 Digitalisierung in der stationären Langzeitpflege - 127

9.3.1 Anwendungsmöglichkeiten - 127

9.3.2 Auswirkungen der Digitalisierung auf die Arbeitsorganisation und Führungskultur - 128

9.4 Shared-Leadership-Ansatz als Führungskonzept - 129

9.4.1 Definition Shared-Leadership-Ansatz - 129

9.4.2 Führungserfolg des Shared-Leadership-Ansatzes - 130

9.4.3 Umsetzung des Shared-Leadership-Ansatzes - 130

9.5 Chancen und Risiken des Shared-Leadership-Ansatzes im Kontext der digitalisierten stationären Langzeitpflege - 131

$9.6 \quad$ Fazit -132

$$
\text { Literatur - } 133
$$




\section{- Zusammenfassung}

In diesem Beitrag werden vor dem Hintergrund der Arbeitsbedingungen in der stationären Langzeitpflege und der Präferenzen der Mitarbeitenden die Möglichkeiten des Einsatzes digitaler Techniken zur Entlastung des Pflegepersonals erörtert. Eine Analyse der Auswirkungen zeigt, dass sich erhebliche Veränderungen in der Arbeitsorganisation ergeben. Um die neuen Bedingungen der Arbeitsorganisation effizient nutzen zu können, ist es zudem erforderlich, die Führungskultur an die Erwartungen der Pflegekräfte anzupassen.

Der Shared-Leadership-Ansatz greift die Wünsche der Pflegekräfte nach Partizipation und Autonomie auf, da alle gemeinsam im Team Führungsverantwortung übernehmen. Wenn die technischen Möglichkeiten so eingesetzt werden, dass die Pflegekräfte in ihrem Handeln unterstützt werden, erscheint diese Führungskultur durch den digitalen Transformationsprozess mehr Möglichkeiten zur Umsetzung in der stationären Langzeitpflege zu bieten. Zur erfolgreichen Umsetzung bedarf es aber eines Umdenkens der Führungskräfte, denn Macht durch direktive Einflussnahme muss abgegeben werden. Dafür sind die Pflegekräfte und die sozialen Beziehungen untereinander zu fördern. In mehreren Studien konnte gezeigt werden, dass mit dem SharedLeadership-Ansatz die Leistungsfähigkeit der Teams gestärkt werden kann. Damit könnten mithilfe digitaler Techniken und der passenden Führungskultur die Arbeitsbedingungen in der stationären Langzeitpflege verbessert werden, was sich wiederum positiv auf die Versorgung der Pflegebedürftigen auswirken würde.

This paper discusses the possibilities of using digital technologies to relieve the burden on nursing staff against the background of working conditions in long-term care for the elderly and the preferences of employees. An analysis of the effects shows that there are considerable changes in the organisation. In addition, in order to make efficient use of the new conditions, it is necessary to adapt the management culture to the expectations of the nursing staff.
The shared leadership approach picks up on the wishes of employees for participation and autonomy, because everyone in the team takes leadership responsibility. If the technical possibilities are used in such a way that the nursing staff are supported in their actions, this management culture appears to offer more opportunities for implementation in long-term care for the elderly through digital transformation. Successful implementation, however, requires the managers to rethink because power must be relinquished through directive influence. To this end, the employees and the social relationships between them must be promoted. Several studies have shown that the shared leadership approach can strengthen team performance. With the use of digital technologies and the appropriate management culture, working conditions in nursing care for the elderly could be improved, which in turn would have a positive effect on the care of those in need of long-term care.

\subsection{Einleitung}

Arbeitsorganisation und Führungskultur beeinflussen die Arbeitsbelastung und die $\mathrm{Zu}$ friedenheit der Pflegekräfte maßgeblich. Allerdings ist festzustellen, dass die Rahmenbedingungen für stationäre Langzeitpflegeeinrichtungen derart hohe Herausforderungen stellen, dass allein durch organisatorische Maßnahmen oder Veränderungen in der Führungskultur die Probleme nicht zu bewältigen sind. Vielfach wird darüber diskutiert, ob technische Entwicklungen zur Unterstützung der Pflege eingesetzt werden können. Der Gesetzgeber sieht hier Potenziale und will mit dem „Pflegepersonal-Stärkungsgesetz" den Einsatz in der stationären Langzeitpflege vorantreiben. Es werden Fördermittel bis zu $12.000 €$ für Investitionen in digitale Techniken, die das Personal in der Pflege unterstützen, zur Verfügung gestellt. Die digitalen Lösungen sollen sowohl die Pflegenden bei ihrer Arbeit entlasten als auch die individuellen Wünsche der Pflegebedürftigen besser erfüllen und somit deren Le- 
bensqualität erhöhen (Schneider et al. 2017, S. 208). Vor diesem Hintergrund beurteilen auch Pflegekräfte die Entwicklung positiv, solange die zwischenmenschliche Arbeit mit den zu Pflegenden nicht beeinträchtigt wird (Merda et al. 2017, S. 144).

Es gibt aber nur wenige Überlegungen da$\mathrm{zu}$, welche Auswirkungen die Digitalisierung auf die Arbeitsorganisation und die Führungskultur haben. In diesem Beitrag wird der Frage nachgegangen, welche Möglichkeiten sich durch die Digitalisierung in der Arbeitsorganisation der stationären Langzeitpflege ergeben können und wie sich dieser Veränderungsprozess auf die Führungskultur auswirkt. Denn es ist davon auszugehen, dass „ein Mehr an Vernetzung und der Einsatz neuer IT-Systeme wenig nutzen, wenn das Führungsverständnis ein traditionelles bleibt" (van Dick et al. 2016). Die Akzeptanz der Pflegekräfte sollte gestärkt werden, indem sie auf dem Weg der Digitalisierung mitgenommen werden und auf den Veränderungsprozess Einfluss nehmen können. Der Shared-Leadership-Ansatz ermöglicht die Teilhabe aller Teammitglieder im Arbeitsprozess und soll daher als ein mögliches Managementinstrument in den veränderten Rahmenbedingungen der stationären Langzeitpflege diskutiert werden.

\subsection{Situation in der stationären Langzeitpflege}

\subsubsection{Strukturelle Bedingungen}

Die Bevorzugung der ambulanten pflegerischen Versorgung hat zur Folge, dass in den Pflegeeinrichtungen der Bedarf an professioneller Pflege und damit die Arbeitsbelastung deutlich gestiegen sind (Schneider et al. 2017, S. 207). Erst wenn die Versorgung in der häuslichen Umgebung nicht mehr sichergestellt werden kann, steht der Umzug in eine stationäre Einrichtung an. Das führt dazu, dass es sich in den Einrichtungen größtenteils um multimor- bide, kognitiv eingeschränkte oder sterbende Pflegebedürftige handelt.

Daneben wirken sich die organisatorischen Rahmenbedingungen ungünstig auf die $\mathrm{Zu}$ friedenheit des Personals aus. Eine Fallanalyse, die von der Autorin im Rahmen des Forschungsprojektes SITA durchgeführt wurde (Menje 2018, S. 8 f.), zeigt sowohl in der quantitativen als auch in der qualitativen Erhebung, dass die Arbeitszeiten in mehrfacher Hinsicht von den Pflegekräften als belastend wahrgenommen werden. Hierzu zählen insbesondere die langen Arbeitsperioden, Wechselschichtdienste und das häufige ungeplante Einspringen bei Ausfall von Kolleginnen und Kollegen. Bei der Überprüfung der Auswirkungen auf das Kohärenzgefühl konnte festgestellt werden, dass die Sinnhaftigkeit der Arbeit und die Sinnhaftigkeit der Dienstzeiten positiv bewertet werden. Dagegen werden die Dimensionen der Handhabbarkeit und Planbarkeit der Arbeitszeiten sehr niedrig bewertet. Somit fehlt den Mitarbeitenden ein Gefühl der Stimmigkeit mit der Arbeit, wodurch teilweise erklärbar ist, dass es zu vermehrten Krankheitsausfällen kommt. Denn das Kohärenzgefühl trägt dazu bei, Widerstandsressourcen zur Bewältigung von Stresssituationen für sich zu nutzen (Blättner 2007, S. 68).

Die schwierigen Arbeitsbedingungen in der Pflege spiegeln sich in hohen Krankheits- und Fluktuationsquoten wider. Mit durchschnittlich 27,2 Krankheitstagen im Jahr 2017 gehört die Altenpflege zu den Berufen, die einen hohen Krankenstand aufweisen (Meyer et al. 2018, S. 353). Eine Auswertung der Routinedaten der Barmer GEK des Jahres 2012 zeigt eine durchschnittliche Fluktuationsquote in der Berufsgruppe von 9\% (Müller und Rothgang 2015, S. 27). Bei einer durchschnittlichen Vakanzzeit von 175 Tagen (Bundesagentur für Arbeit 2018, S. 18) steigen die Belastungen für die verbleibenden Mitarbeitenden. Ein Teufelskreis, aus dem es auszubrechen gilt.

Die Bundesregierung sieht die zunehmende Problematik und finanziert mit einem Sofortprogramm insgesamt 13.000 Pflegekraftstellen in stationären Langzeitpflegeeinrichtungen. An 


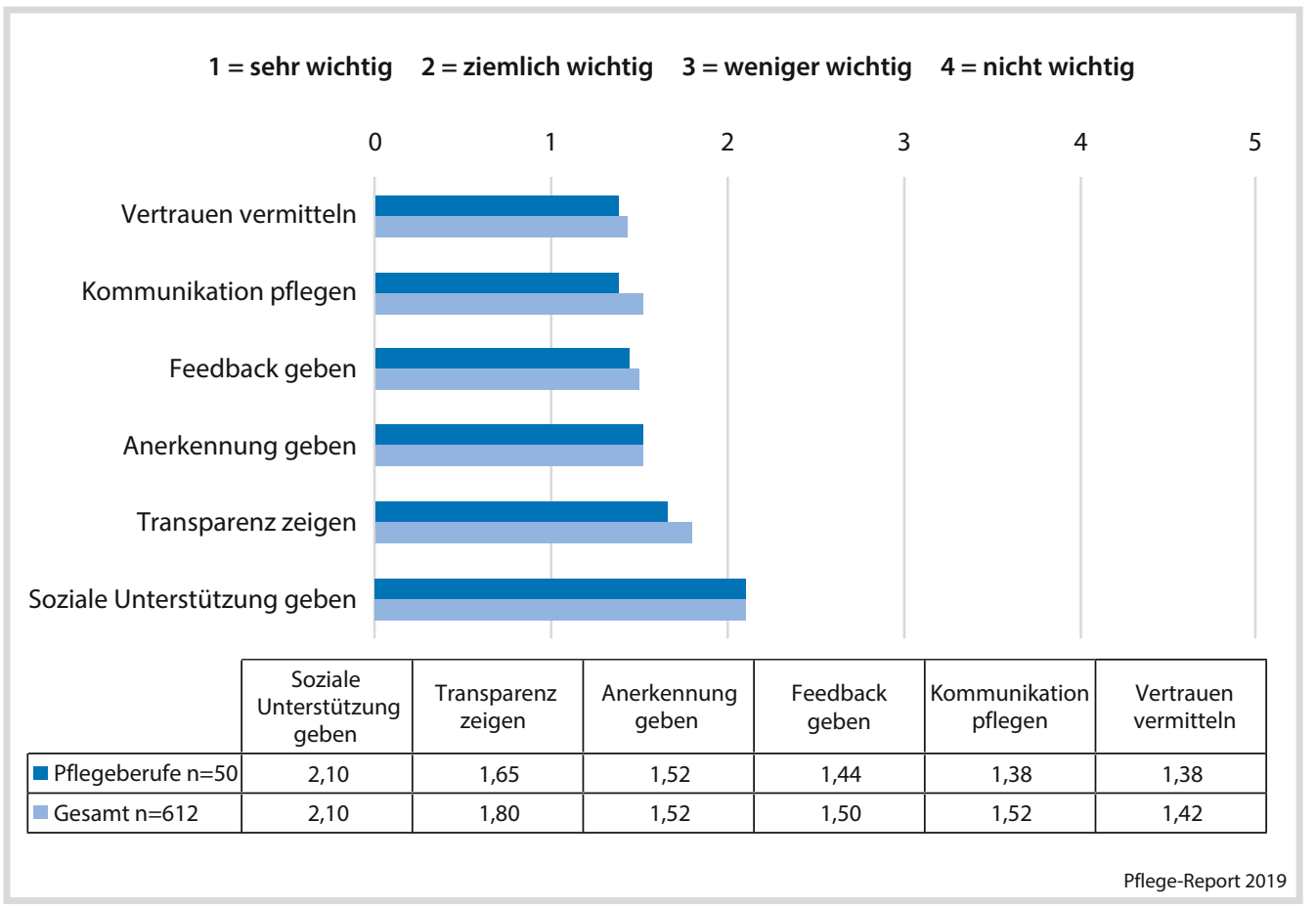

- Abb. 9.1 Wie wichtig sind Ihnen folgende Verhaltenseigenschaften in der Personalführung Ihres Vorgesetzten in einem potenziellen Unternehmen? (Gesamt $n=612$, Pflegeberufe: $n=50$ )

zweiter Stelle dieses Programms steht die Digitalisierung. Es wird davon ausgegangen, dass diese, wenn sie „richtig eingesetzt [wird], ein erhebliches Potenzial zur Entlastung der Pflegekräfte" hat (Bundesministerium für Gesundheit $2019^{1}$ ). Da Investitionen in die Digitalisierung und Technisierung in Zukunft durch die Pflegeversicherung kofinanziert werden, ist davon auszugehen, dass die Technik zunehmend Einzug in den Alltag der Pflegeeinrichtungen nehmen wird.

\subsubsection{Präferenzen der Pflegekräfte}

Pflegekräfte, die mit hohem Idealismus in diesen Beruf gestartet sind und Freude an dem Umgang mit den Pflegebedürftigen und der Arbeit im Team haben, werden von der Realität

1 https://www.bundesgesundheitsministerium.de/ sofortprogramm-pflege.html. enttäuscht. Unter Zeitdruck sind die Aufgaben, die sie per Anordnung aufgetragen bekommen, zu erfüllen. Vielfach sind Leitungskräfte überlastet, da sie ein Großteil ihrer Arbeitszeit damit verbringen, den Mangel an Personal aufzufangen. Vertrauen untereinander, respektvoller Umgang miteinander, eine offene und transparente Kommunikation bleiben oftmals auf der Strecke. Aber dies sind gerade die Werte, die die Pflegekräfte besonders hoch schätzen. Frauen und Männer möchten gleichberechtigt und selbstbestimmt arbeiten sowie Beruf, Familie und Freizeit miteinander vereinbaren (Rösler et al. 2018, S. 9).

In einer Online-Befragung der Autorin im Jahr 2016 mit 612 Teilnehmerinnen und Teilnehmern (Durchschnittsalter 28 Jahre), wobei 50 Personen im Pflegeberuf tätig sind, haben bei der Frage „Wie wichtig sind Ihnen folgende Verhaltenseigenschaften in der Personalführung Ihres Vorgesetzten in einem potenziellen Unternehmen?“ $60 \%$ (64\% der Pflegekräfte) es 
als sehr wichtig angesehen, dass ihnen die Führungskraft Vertrauen entgegenbringt. Zudem ist es wichtig, Feedback und Anerkennung zu bekommen. Außerdem wird viel Wert auf die Kommunikation gelegt. Ziemlich wichtig ist es, Transparenz und soziale Unterstützung zu haben - Abb. 9.1.

Wie oben bereits dargestellt, wird sich für die Pflegekräfte in der stationären Langzeitpflege in der nahen Zukunft durch die finanzielle Förderung der Digitalisierung eine weitere Dimension auftun. Bisher sind die Einstellungen der Pflegekräfte hierzu noch nicht umfassend bekannt, da es noch viele unbekannte Faktoren gibt. Geht man davon aus, dass die Ergebnisse einer Online-Befragung mit 495 Teilnehmerinnen und Teilnehmern zu den Wertewelten (Rösler et al. 2018, S. 17) auf die Gruppe der Pflegenden in der stationären Langzeitpflege übertragbar sind, dann befürchten $85 \%$, dass mit zunehmender Digitalisierung auch die Kontrolle zunehmen wird. Diese Annahme wird durch das Ergebnis der Studie von Brown et al. (2010) unterstützt. Die quantitative und qualitative Untersuchung in drei britischen Pflegeeinrichtungen zeigt, dass mit IT-basierten Dokumentationssystemen das organisationale Commitment sinkt, da die Arbeitsleistungen vermehrt kontrolliert werden (Brown et al. 2010). Hülsken-Giesler stellt fest, dass grundsätzlich ein spannungsreiches und ambivalentes Verhältnis der Pflege zur Technik besteht (Hülsken-Giesler 2010, S. 308). Um dies nachvollziehen zu können, wird im folgenden Abschnitt ein Überblick darüber gegeben, in welchen Bereichen digitale Techniken Pflegetätigkeiten in der stationären Langzeitpflege verändern können.

\subsection{Digitalisierung in der stationären Langzeitpflege}

\subsubsection{Anwendungsmöglichkeiten}

Aus der Vielfalt der digitalen Entwicklungen ergeben sich vier Organisationsprinzipi- en: technische Assistenz, Informationstransparenz, dezentrale Entscheidungen und Vernetzung (Ralfs 2018, S. 62).

Mit der technischen Assistenz kann das Pflegepersonal von Tätigkeiten entlastet werden oder es können Aufgaben schneller erledigt werden. Damit werden Arbeitsprozesse verschlankt. Hierzu zählen z. B. Service- und Transportroboter, die Pflegeutensilien wie etwa Wäsche automatisch zum Einsatzort bringen (Daum 2017, S. 24; Graf et al. 2018, S. 29). Aber auch reine Pflegetätigkeiten wie die Positionierung des Pflegebedürftigen können von der Technik erledigt werden: Intelligente Matratzen mit Drucksensoren verändern die Liegeposition der Pflegebedürftigen selbständig und verhindern somit, dass Druckgeschwüre entstehen (Munstermann 2015, S. 44).

Mit der Informationstransparenz können Tätigkeiten zur Erfassung von Daten reduziert werden, die Professionalität des Handelns gefördert und Arbeitsprozesse optimiert werden. Beispielsweise können Vitalparameter oder Stürze automatisch mit Sensoren erfasst und an die Pflegekräfte weitergegeben werden (Schneider et al. 2017, S. 217 f.; Munstermann 2015, S. 44; 46). Dokumentationstätigkeiten können schneller durchgeführt werden, wenn mithilfe der Spracherfassung Pflegeinformationen in den Bewohnerzimmern oder mit mobilen Telefonen aufgenommen werden und dann automatisch in die Pflegedokumentation einfließen (Becker 2019, www.arbeitenviernull. de/). Fachinformationen, mit denen die Professionalität des Pflegehandelns verbessert werden kann, können den Pflegekräften jederzeit über in Brillen integrierte Bildschirme oder am Handgelenk getragene Displays (Smartwatch) mobil zur Verfügung gestellt werden (Daum 2017, S. 21; Kreidenweis 2018, S. 19). Arbeitsprozesse können optimiert werden, indem an Tätigkeiten wie z. B. die Medikamentenverabreichung erinnert wird (Daum 2017, S. 21).

Die Möglichkeit, dezentral Entscheidungen treffen zu können, wird dadurch geschaffen, dass jeder in einem Entscheidungsprozess $\mathrm{Zu}$ gang zu den Informationen hat. So kann z. B. der Dienstplanungsprozess auf das Team über- 
tragen werden, wenn die Teammitglieder einen digitalen Zugang zum aktuellen Planungsstand haben. Zudem können Informationen so aggregiert, visualisiert und verständlich aufbereitet werden, dass auf dieser Basis fundiert Entscheidungen getroffen werden können (Scheuer 2017, S. 315). Darüber hinaus können intelligente digitale Systeme aus Informationen lernen und selber Lösungen entwickeln (Mainzer 2016, S. 3; Daum 2017, S. 25). Beispielsweise können Arbeitsprozesse auf Basis der in den digitalen Pflegeakten erfassten Daten über Pflegebedarfe und die zur Verfügung stehenden Personalressourcen optimiert geplant und das Personal effizient eingesetzt werden (Rösler et al. 2018, S. 25).

Vernetzte digitale Systeme können außerdem Informationen aktuell, ortsunabhängig und transparent bereitstellen. Damit lässt sich die inter- und intraprofessionelle Vernetzung, z. B. mit dem Hausarzt, deutlich einfacher fördern (Merda et al. 2017, S. 144). Zudem eröffnen sich neue Möglichkeiten der Arbeitsorganisation, denn auf diese Daten kann mobil zugegriffen werden, sodass z. B. Überwachungsarbeiten am Bildschirm oder planerische und organisatorische Aufgaben nicht vor Ort in der Pflegeeinrichtung durchgeführt werden müssen.

\subsubsection{Auswirkungen der Digitalisierung auf die Arbeitsorganisation und Führungskultur}

Digitalisierung in der Arbeitswelt führt zu tiefgreifenden Veränderungen in der Arbeitsorganisation und in den Berufsfeldern (Korff 2018, S. 181). Arbeitsplätze und ihre Aufgaben verändern sich, sodass Strukturen und Prozesse, die die Rahmenbedingungen der technisierten Arbeitswelt sind, infrage zu stellen und anzupassen sind.

Zum einen verlagert sich Arbeit von der Erfassung der Informationen bei den Pflegebedürftigen auf das kritische Bewerten der di- gital erfassten Daten, der entwickelten Lösungen und das Anstoßen neuer digitaler Prozesse. Diese Entwicklung ist mit der Sorge bei den Pflegekräften verbunden, dass die eigentliche Arbeit am Pflegebedürftigen in den Hintergrund tritt. Fachliche Kompetenzen, die sich darauf beziehen, pflegerisches Wissen parat zu haben und aus pflegerelevanten Informationen Schlussfolgerungen abzuleiten, können durch künstliche Intelligenz unterstützt werden. Zum anderen wird die körperliche Arbeit der Pflegekräfte, die das Muskel-Skelett-System belastet, durch technische Assistenzsysteme entlastet.

Die digitale Vernetzung untereinander sowie mit internen und externen Ressourcen ermöglicht eine neue Art der Kommunikation. Die Pflegeeinrichtung selbst stellt keine Grenze mehr dar, auch darüber hinaus ist eine grenzenlose Kommunikation möglich. Durch die Vernetzung haben alle Pflegekräfte schnell und zeitgleich den gleichen Wissensstand. Dadurch können sie ortsunabhängig aktiv an Arbeitsund Entscheidungsprozessen mitwirken. Damit kann in der Arbeitsorganisation Flexibilität geschaffen werden und die Pflegekräfte haben bei der Erledigung beispielsweise von Verwaltungsaufgaben mehr Freiräume. Die Folge ist, dass Arbeitsplätze mit ihren Anforderungen und Aufgaben neu durchdacht werden müssen.

Ein erfolgreicher Transformationsprozess setzt aber voraus, dass in der Belegschaft und bei den Pflegebedürftigen eine grundlegende Zufriedenheit und Akzeptanz für die Digitalisierung hergestellt wird. Dabei darf nicht übersehen werden, dass zum einen Ängste vor den Kontrollmöglichkeiten bestehen und sich zum anderen die Ansprüche der Mitarbeitenden und der Bewohnerinnen und Bewohner verändern, denn die vernetzte Welt führt möglicherweise dazu, dass schnelle und flexible Reaktionen von den Pflegekräften und den Führungskräften erwartet werden.

Außerdem sind die Entwicklungen rasant, sodass eine stetige Veränderungsbereitschaft ein wichtiges Attribut geworden ist. Hierarchische Strukturen und starre Organisationspro- 
zesse sowie traditionelle Führungsstile können da nicht mehr den notwendigen Rahmen bieten und gelten als veraltet (Teichmann und Hüning 2018, S. 27; Peters 2017, S. 25). Die Mitarbeitenden müssen die Möglichkeiten bekommen, in den Veränderungsprozess eingreifen zu können, damit die Ängste vor den Veränderungen überwunden werden und eine Offenheit gegenüber neuen Aufgaben geschaffen wird.

Planen, Organisieren, Informieren oder Kontrollieren sind Kernaufgaben der Führung und werden vielfach in der Praxis auch als solche angesehen. Durch diese Tätigkeiten wird einer Führungskraft Macht verliehen; sie entscheidet, wer was wie macht und ob es den Anforderungen entspricht. Hierfür gibt es nun IT-Systeme, die schneller auf der Basis einer umfassenden Informationslage solche Aufgaben übernehmen können. Das bedeutet, dass die digitale Transformation mit einem Machtverlust bei den Führungskräften einhergehen kann. Aufgabe der Führungskraft sollte mit zunehmender Digitalisierung sein, den Pflegekräften die digitalen Ressourcen zur Verfügung zu stellen und sie in ihren Fähigkeiten zu unterstützen, die Informationen $\mathrm{zu}$ erfassen, zu verarbeiten und in den Arbeitsprozess einfließen zu lassen. Geschieht dies nicht, sondern werden die digitalen Informationen zur Ausweitung der Kontrolle der Mitarbeitenden genutzt, werden mögliche Potenziale der IT nur schwer zu nutzen sein.

Deutlich wird: Die digitale Transformation zieht nicht nur einen technischen Wandel in der Pflege nach sich, sondern erfordert darüber hinaus in den Pflegeeinrichtungen einen Kultur- und Führungswandel. Im Rahmen dieses Wandels muss berücksichtigt werden, dass einerseits Ängste und Vorbehalte der Pflegekräfte gegenüber der Technisierung bestehen, zum anderen die Entlastung von Aufgaben sowie die Möglichkeiten, pflegerisch kompetent zu handeln, durch technische Entwicklungen unterstützt werden. Dadurch können die Führungskräfte entlastet werden, indem den Pflegekräften Verantwortung übertragen wird. Dies hat zudem den Vorteil, dass durch die
Einflussnahme der Pflegekräfte auf Entscheidungen die Sorge hinsichtlich der Kontrolle reduziert werden kann. Ein Führungskonzept, das diese Überlegungen aufnimmt, ist der Shared-Leadership-Ansatz, der im Folgenden vorgestellt und hinsichtlich des Führungserfolges untersucht wird.

\subsection{Shared-Leadership-Ansatz als Führungskonzept}

\subsubsection{Definition Shared-Leadership-Ansatz}

Das hervorstechende Merkmal des SharedLeadership-Ansatzes ist, dass nicht die Führungsperson in ihren Eigenschaften, ihrem Verhalten oder ihrer Interaktion im Mittelpunkt des Interesses steht. Von diesem klassischen Führungsverständnis der vertikalen Führung kann die geteilte Führung abgegrenzt werden, da diese direkt aus dem Team hervorgeht und auf einer Ebene kollegial stattfindet (Piecha et al. 2012, S. 560). Ausgehend von den gesellschaftlichen Erwartungen der Mitarbeitenden, in Entscheidungsprozesse eingebunden zu werden, hat dieser Führungsansatz an Bedeutung gewonnen (Lang und Rybnikova 2014, S. 152).

In der Literatur wird der Shared-Leadership-Ansatz definiert als eine gegenseitige Beeinflussung und gemeinsame Verantwortung der Teammitglieder, wobei sie sich gegenseitig zur Zielerreichung führen (Wang et al. 2014, S. 181). Zu finden ist er dann, wenn in einer Gruppe die Funktionen der Führung auf die Gruppenmitglieder verteilt sind (Drescher et al. 2014, S. 772). Die Führung wird unter mehreren teilnehmenden Personen verteilt und geteilt, anstatt sie von einer einzelnen Person ausführen zu lassen (Meuser et al. 2016, S. 1.390). Führung findet auf der Gruppenebene statt, die auf gegenseitigem Vertrauen und gemeinsamer Einflussnahme unter den Teammitgliedern basiert, um Teamziele zu erreichen (Chiu et al. 2016, S. 1.705). Es ist ein dy- 
namischer Führungsansatz, denn verschiedene Personen des Teams können im Laufe der Zeit zu unterschiedlichen Zeitpunkten Führungsrollen und Followerrollen ausüben (Lord et al. 2017, S. 444). Aus den unterschiedlichen Definitionen lassen sich die drei Hauptmerkmale des Shared-Leadership-Ansatzes ableiten: Die Teammitglieder führen sich gegenseitig, es handelt sich um emergente Teamstrukturen und Führungsrollen. Zudem ist die Einflussnahme über die Teammitglieder verteilt (Zhu et al. 2018, S. 835).

Der Shared-Leadership-Ansatz sieht demnach vor, dass sowohl die Führungskraft als auch die Mitarbeitenden Führungsaufgaben übernehmen, wobei der Führungskraft die besondere Funktion des „Katalysators“ zukommt (Schirmer und Woydt 2016, S. 195 f.). Es werden aber nicht nur die Aufgaben aufgeteilt, sondern die Mitarbeitenden übernehmen zudem Führungsverantwortung für die Zielerreichung. Damit geht einher, dass Macht und die Möglichkeiten der Einflussnahme aufgeteilt werden (Lang und Rybnikova 2014, S. 151). Zum Beispiel können Teammitglieder, die in einem speziellen Bereich qualifiziert sind, in diesem Bereich die Führung übernehmen, während sie die Rolle des Mitarbeiters in anderen Bereichen einnehmen (Zhu et al. 2018, S. 835).

\subsubsection{Führungserfolg des Shared- Leadership-Ansatzes}

Studien zeigen unter anderem, dass der SharedLeadership-Ansatz langfristiger und stabiler zum Führungserfolg führt, da Führungskräfte nicht die Möglichkeit haben, egoistisch Ziele durchzusetzen und $\mathrm{zu}$ polarisieren, was von den Mitarbeitenden positiv aufgenommen wird (Furtner 2017, S. 22).

Analysen haben zudem ergeben, dass die geteilte Führung die Effektivität der Teams erhöht. Die Zufriedenheit mit der Arbeit, die Motivation im Team sowie das Vertrauen untereinander steigen, was sich positiv auf die Leistung auswirkt. Zudem wird dadurch, dass alle am Führungsprozess teilnehmen und somit ein Austausch von Informationen stattfindet, "ein weitreichendes transaktives Wissenssystem" geschaffen (Piecha et al. 2012, S. 563). Allerdings kann es zu Verzögerungen in Entscheidungsprozessen kommen oder das Team wird orientierungslos. Zudem kann die gegenseitige Führung zur destruktiven Machtausübung genutzt werden (Schirmer und Woydt 2016, S. 197; Wegge und Rosenstiel 2014, S. 355 ff.)

Der Führungserfolg zeigt sich laut empirischer Studien darin, dass der SharedLeadership-Ansatz den Zusammenhalt in Gruppen erhöht und freiwillige Mehrleistungen der Mitarbeitenden fördert. Die Problemlösequalität und die Gruppeneffektivität kann gesteigert werden (Pearce und Sims 2002; Bergman et al. 2012). Eine höhere Teamleistung konnten auch Wang et al. (2014) in ihrer Metaanalyse von 42 empirischen Studien nachweisen. Außerdem konnte beobachtet werden, dass vermehrt innovatives Verhalten gezeigt wird (Hoch und Kozlowski 2014). Die Beziehungen der Teammitglieder untereinander verbessern sich, denn es gibt weniger Konflikte, es besteht eine höhere Bereitschaft der Konsensfindung und ein stärkeres Vertrauen untereinander (Bergman et al. 2012). Da keine Studien identifiziert werden konnten, in denen die Untersuchung in der stationären Langzeitpflegeeinrichtung stattgefunden hat, werden im Folgenden die Bedingungen zur Umsetzung erörtert.

\subsubsection{Umsetzung des Shared- Leadership-Ansatzes}

Dieser Führungsansatz kann nicht unabhängig von grundsätzlichen organisatorischen und unternehmenskulturellen Rahmenbedingungen gesehen werden (Schirmer und Woydt 2016, S. 196). Eine förderliche Unternehmenskultur in einer Pflegeeinrichtung liegt vor, wenn die Mitarbeitenden ähnliche Vorstellungen über Ziele und deren Erreichung haben, sich die Teammitglieder sozial gegen- 
seitig unterstützen und die Möglichkeiten des Einzelnen, auf Entscheidungen Einfluss zu nehmen, vorhanden sind (Avolio et al. 2009, S. 432; Carson et al. 2007). Außerdem sollte Selbständigkeit und Verantwortungsübernahme von den Teammitgliedern erwünscht sein sowie unterschiedliche Ansichten als wertvoll angesehen werden. Geteilte Führung lässt sich insbesondere dann umsetzen, wenn die Aufgaben komplex sind und verschiedene Kompetenzen verlangen. Dies trifft für Pflegeeinrichtungen $\mathrm{zu}$, denn die Versorgung Pflegebedürftiger ist komplex, da die Bedürfnisse der einzelnen ganz unterschiedlich und wechselnd sind. Diese sind von den Pflegekräften zu erkennen und die notwendigen Pflegehandlungen sind abzuleiten und umzusetzen.

Allerdings sind Aufgaben, bei denen schnelle Entscheidungen notwendig sind, zumeist weniger geeignet. Das würde heißen, dass in medizinischen Notfallsituationen dieses Führungskonzept an seine Grenzen stößt. Geteilte Führung ist zudem nur dann möglich, wenn die Kompetenzen bei den Teammitgliedern vorhanden sind. Hierbei handelt es sich sowohl um die fachlichen Kompetenzen, die zur Erfüllung der Aufgaben benötigt werden, als auch um die Führungskompetenzen (Piecha et al. 2012, S. 564 f.). Da in Pflegeeinrichtungen nicht nur Fachkräfte eingesetzt werden, könnte dies in der praktischen Umsetzung problematisch sein. Es ist dennoch vorstellbar, dass im Digitalisierungsprozess durch die Möglichkeiten der digitalen Wissensbereitstellung Hilfskräfte für bestimmte Aufgabenbereiche Kompetenzen erlangen, in denen sie eigenverantwortlich handeln können.

Die Positionsmacht der Leitungskräfte wird im Shared-Leadership-Ansatz nicht dazu genutzt, die eigenen oder die unternehmerischen Interessen durch- und umzusetzen. Die Leitungskraft dient dazu, die Teammitglieder zu unterstützen und $\mathrm{zu}$ entwickeln. Grundlage hierfür ist ein hohes Ausmaß an Selbstkontrolle und ein sozialisiertes Machtmotiv. Für eine erfolgreiche Umsetzung bedarf es der Überprüfung, inwieweit die Leitungskräfte dieses mitbringen oder eventuell mit Schulungen geför- dert werden sollten. Zur Zufriedenheit bei den Leitungskräften führt dieses Vorgehen dann, wenn die Geführten der Leitungskraft Wertschätzung und Dankbarkeit entgegenbringen (Furtner 2017, S. 22).

Der Shared-Leadership-Ansatz lässt sich im Führungsverhalten folgendermaßen umsetzen: Zum einen wird die Aufgabenzuweisung auf die Gruppe übertragen, indem Aufgaben, z. B. wer welche Bewohner pflegt oder wer welche pflegerischen Aufgaben übernimmt, gemeinsam verteilt werden. Hierzu gehört, dass die zur Aufgabenerfüllung notwendigen Informationen zur Verfügung stehen und die Erwartungen an die Aufgabenerfüllung klar kommuniziert werden. Zum anderen sind die Beziehungen der Mitglieder im Pflegeteam zu fördern, indem der Zusammenhalt gestärkt wird. Hierbei ist aber auch darauf zu achten, dass jeder einzelne mit seinen Leistungen anerkannt wird und die Bedürfnisse und Anliegen der einzelnen Mitglieder wahrgenommen werden. Außerdem ist die gemeinsame weitere Entwicklung des Pflegeteams zu unterstützen, indem Veränderungen und neue Anforderungen erfasst und gemeinsam - unter Einbeziehungen der bisherigen Erfahrungen - Handlungsmöglichkeiten entwickelt werden. Dies ist z. B. dann der Fall, wenn neue, digitale Pflegetechniken wie oben beschrieben Einzug in den Pflegealltag halten. Intern sollte die Führungskraft dafür sorgen, dass die Kommunikation mit Hilfe der digitalen Techniken für alle Teammitglieder transparent ist und extern sollte sie das Team nach außen hin vertreten (Kauffeld et al. 2017, S. 235 f.).

\subsection{Chancen und Risiken des Shared-Leadership-Ansatzes im Kontext der digitalisierten stationären Langzeitpflege}

Durch den Fachkräftemangel in der stationären Langzeitpflege und den demografischen Wandel wird es unumgänglich sein, neue Wege zu gehen, um die Herausforderungen der Zukunft 
meistern zu können. Damit die Arbeit überhaupt noch leistbar bleibt, sollte Offenheit für den Einsatz technischer Möglichkeiten in der Pflege geschaffen werden. Die Chancen, die sich durch die Erfassung und Verarbeitung von Informationen ergeben, sind nicht zu übersehen. Standardisierte Aufgaben können von der Technik übernommen werden und komplexere Aufgaben lassen sich einfacher und schneller lösen, da die erfassten Informationen ausgewertet und verarbeitet werden. Damit können Arbeitsprozesse in Pflegeeinrichtungen effizienter gestaltet und umgesetzt werden.

Dies wird aber nur gehen, wenn die Menschen - sowohl die Pflegebedürftigen als auch die Pflegekräfte - mit ihren Bedürfnissen, Interessen und Ängsten in den digitalen Transformationsprozess einbezogen werden. Die Ängste, die sich durch die Erhebung der Daten und die damit verbundenen Sorgen ergeben, sind hierbei besonders ernst zu nehmen. Es besteht die Gefahr, dass die Beschäftigten unter dauerhafter Kontrolle sind, denn alles kann erfasst und ausgewertet werden. Wichtig ist es, die Wünsche der Pflegekräfte wie Selbstbestimmtheit und Vertrauen zu berücksichtigen. Geschieht dies nicht, ist zumeist Unzufriedenheit mit dem Arbeitsplatz oder sogar mit dem Beruf die Folge.

Neben der digitalen Transformation ist es demnach notwendig, das Führungskonzept kritisch zu hinterfragen. Der SharedLeadership-Ansatz könnte wertvolle Ansätze bieten: Pflegekräfte übernehmen gemeinsam die Führungsaufgaben. Dies könnte unter den neuen Rahmenbedingungen auch in der stationären Langzeitpflege umsetzbar sein. Allen Pflegekräften stehen die notwendigen Informationen zur Verfügung, um fachlich adäquat handeln zu können. Die Pflegekräfte können auf Entscheidungshilfen durch eine analytische Aufbereitung der Daten zurückgreifen. Somit können Entscheidungen dezentral getroffen werden. Außerdem kann die fachliche Kompetenz durch Bereitstellung der Informationen unterstützt werden.

Die Mitarbeitenden haben somit Zugang $\mathrm{zu}$ allen Informationen und nehmen partizipa- tiv an Entscheidungsprozessen teil. Dies führt zu einer höheren Akzeptanz und wie nachgewiesen zu einer höheren Teamleistung. Zudem kann das Kohärenzgefühl der Pflegekräfte gestärkt werden, denn durch die Einflussnahme auf die Gestaltung der Arbeitsprozesse kann das Gefühl der Planbarkeit und Vorhersehbarkeit gesteigert werden. Dadurch werden Ressourcen aufgebaut, die ihre Gesunderhaltung in Stresssituationen fördern.

Dennoch darf nicht übersehen werden, dass die Gefahr der Zunahme von Konflikten besteht, z. B. wenn gemeinsam Verantwortung für die Dienstplangestaltung übernommen wird. Zudem kann die Konsensbildung, z. B. über Pflegeprozesse, ein zu langwieriger und ressourcenverbrauchender Prozess sein. Die Aufgaben der Führungskraft werden sich dementsprechend ändern. Direktive Durchsetzung durch den Einsatz von Machtmitteln ist weder nötig noch möglich. Die Herausforderungen werden in der Förderung der sozialen Beziehungen im Team und der Zusammenführung zur gemeinsamen Zielsetzung liegen.

\subsection{Fazit}

Die aktuellen Arbeitsbedingungen in der stationären Langzeitpflege entsprechen in vielfacher Hinsicht nicht den Wünschen der Pflegekräfte. Selbstständiges Arbeiten, gegenseitiges Vertrauen, die Vereinbarkeit von Familie und Beruf bleiben aufgrund der hohen Arbeitsbelastung auf der Strecke. Der Digitalisierungsprozess bietet die Chance, die Arbeitsbedingungen zu verbessern und professionell Pflegende zu entlasten. Somit entstehen Arbeitsplätze mit anderen Anforderungen an die Mitarbeiterinnen und Mitarbeiter und die Führungskräfte. Sie bekommen Unterstützung, aber die Erwartungen, etwa an die Schnelligkeit und Flexibilität, nehmen zu. Seitens der Pflegekräfte bestehen zudem Befürchtungen, dass die technischen Möglichkeiten zur Kontrolle und nicht zur Entlastung eingesetzt werden.

Aus diesem Grund kann der Digitalisierungsprozess nicht unabhängig von der Füh- 
rungskultur gesehen werden - diese muss der Entwicklung angepasst werden. Da die Pflegekräfte mithilfe der Technik in ihrer Kompetenz unterstützt werden, können sie sich mit mehr Autonomie und Entfaltungsmöglichkeiten in den Arbeitsprozess einbringen. Die Führungskräfte können diese Entwicklung fördern, wenn sie dem Shared-Leadership-Ansatz entsprechend die Führungsverantwortung auf das Team übertragen. Dieses dem Pflegepersonal entgegenbrachte Vertrauen stärkt das Team und führt zu einer höheren Leistungsqualität. Dadurch kann die Sorge vor der Kontrolle abgebaut werden und die Zufriedenheit der Pflegekräfte steigen, wovon wiederum die Pflegebedürftigen profitieren.

\section{Literatur}

Avolio BJ, Walumbwa FO, Weber TJ (2009) Leadership: current theories, research, and future directions. Annu Rev Psychol 60:421-449

Becker W (2019) Sprachsteuerung in der MenschMaschine-Interaktion, Intelligente Vernetzung für Altenpflegedokumentationssysteme: Sprint-Doku. https://www.arbeitenviernull.de/ experimentierraeume/gefoerderte-projekte/inqaexperimentierraeume/sprint-doku/. Zugegriffen: 5. Febr. 2019

Bergman JZ, Rentsch JR, Small EE, Davenport SW, Bergman SM (2012) The shared leadership process in decision-making team. J Soc Psychol 152:17-42

Blättner B (2007) Das Modell der Salutogenese, Eine Leitorientierung für die berufliche Praxis. Präv Gesundheitsf 2:67-73. https://doi.org/10.1007/s11553-0070063-3

Brown K, Korczynski M (2010) When caring and surveillance technology meet. Organizational commitment and discretionary effort in home care work. Work Occup 3:404-432

Bundesagentur für Arbeit (2018) Fachkräfteengpassanalyse, Berichte: Blickpunkt Arbeitsmarkt, Juni 2018. Bundesagentur für Arbeit, Statistik/Arbeitsmarktberichterstattung, Nürnberg

Bundesministerium für Gesundheit (2019) Sofortprogramm Pflege. Gesetz zur Stärkung des Pflegepersonals (Pflegepersonal-Stärkungsgesetz - PpSG). https://www.bundesgesundheitsministerium.de/ sofortprogramm-pflege.html. Zugegriffen: 19. Jan. 2019

Carson JB, Tesluk PE, Marrone JA (2007) Shared leadership in teams: an investigation of antecedent con- ditions and performance. Acad Manage J 50:12171234

Chiu CC, Owens BP, Tesluk PE (2016) Initiating and utilizing shared leadership in teams: the role of leader humility, team proactive personality, and team performance capability. J Appl Psychol 101:1705-1720

Daum M (2017) Digitalisierung und Technisierung der Pflege in Deutschland. Aktuelle Trends und ihre Folgewirkungen auf Arbeitsorganisation, Beschäftigung und Qualifizierung. DAA Stiftung, Hamburg

Van Dick R, Helfritz KH, Stickling E, Gross M, Holz F (2016) Digital Leadership - die Zukunft der Führung in Unternehmen. https://www.dgfp.de/fileadmin/user_ upload/DGFP_e.V/Medien/Publikationen/20122016/Digital_Leadership_Studie.pdf. Zugegriffen: 21. Nov. 2018

Drescher MA, Korsgaard MA, Welpe IM, Picot A, Wigand RT (2014) The dynamics of shared leadership: Building trust and enhancing performance. J Appl Psychol 99:771-783

Furtner M (2017) Empowering Leadership. Mit selbstverantwortlichen Mitarbeitern zu Innovation und Spitzenleistungen. Springer, Wiesbaden

Graf B, King RS, Rößner A, Schiller C, Ganz W, Bläsing D, Fischbach J, Warner N, Bornewasser M (2018) Entwicklung eines intelligenten Pflegewagens zur Unterstützung des Personals stationärer Pflegeeinrichtungen. In: Pfannstiel MA, Krammer S, Swoboda W (Hrsg) Digitale Transformation von Dienstleistungen im Gesundheitswesen IV, Impulse für die Pflegeorganisation. Springer Gabler, Wiesbaden, S 25-50

Hoch JE, Kozlowski WJ (2014) Leading virtual teams: Hierarchical leadership, structural supports, and shared team leadership. J Appl Psychol 99:390-403

Hülsken-Giesler M (2010) Technikkompetenzen in der Pflege. Anforderungen im Kontext der Etablierung neuer Technologien in der Gesundheitsversorgung. Pflege Ges 15(4):330-352

Kauffeld S, Sauer N, Handke L (2017) Shared Leadership. Gruppe Interakt Organisation 48:235-238. https:// doi.org/10.1007/s11612-017-0381-7

Korff M (2018) Warum sollten Stationen digitalisiert werden? In: Bamberg C, Kasper N, Korff M, Herbold R (Hrsg) Moderne Stationsorganisation im Krankenhaus. Springer, Berlin, S 179-189

Kreidenweis H (2018) Sozialwirtschaft im digitalen Wandel. In: Kreidenweis H (Hrsg) Digitaler Wandel in der Sozialwirtschaft, Grundlagen, Strategien, Praxis. Nomos, Baden-Baden, S 11-26

Lang R, Rybnikova I (2014) Aktuelle Führungstheorien und -konzepte. Springer Gabler, Wiesbaden https:// doi.org/10.1007/978-3-8349-3729-2_6

Lord RG, Day DV, Zaccaro SJ, Avolio BJ, Eagly AH (2017) Leadership in applied psychology: three waves of theory and research. J Appl Psychol 102:434-451

Mainzer K (2016) Künstliche Intelligenz - Wann übernehmen die Maschinen? Springer, Berlin, Heidelberg 
Menje N (2018) Innovationen in der Pflege. Interview mit Christiansen $M$ und Stüber K. sgp. REPORT 19/2018:8-9

Merda M, Schmidt K, Kähler B (2017) Pflege 4.0 - Einsatz moderner Technologien aus der Sicht professionell Pflegender. Forschungsbericht, Stand 8/2017. Berufsgenossenschaft für Gesundheitsdienst und Wohlfahrtspflege (BGW), Hamburg

Meuser JD, Gardner WL, Dinh JE, Hu J, Liden RC, Lord RG (2016) A network analysis of leadership theory: the infancy of integration. J Manage 42:1374-1403

Meyer M, Wenzel J, Schenkel A (2018) Krankheitsbedingte Fehlzeiten in der deutschen Wirtschaft im Jahr 2017. In: Badura B, Ducki A, Schöder H, Klose J, Meyer M (Hrsg) Fehlzeiten-Report 2018. Springer, Berlin Heidelberg, S 331-387

Müller R, Rothgang H (2015) Bedarfs- und Angebotsanalyse und -prognose über Ausbildungsplätze in der Stationäre Langzeitpflegeausbildung, Gutachten im Auftrag der Senatorin für Soziales, Kinder, Jugend und Frauen in Bremen. http://www.socium. uni-bremen.de/uploads/News/2015/150212 Gutachten_StationäreLangzeitpflegeausbildung_ Bericht.pdf. Zugegriffen: 8. Nov. 2018

Munstermann M (2015) Technisch unterstützte Pflege von morgen, Innovative Aktivitätserkennung und Verhaltensermittlung durch ambiente Sensorik. Springer Vieweg, Wiesbaden

Pearce CL, Sims HP (2002) Vertical versus shared leadership as predictors of the effectiveness of change management teams: An examination of aversive, directive, transactional, transformational, and empowering leader behaviors. Group Dyn Theory Res Pract 6:172-197

Peters B (2017) Leadership Agility und Digitalisierung in der Krankenversicherung - Steigende Komplexität und wachsende Dynamik der Digitalisierung erfordern zunehmend agile Organisationen und agile Führungskräfte. In: Pfannstiel MA, Da-Cruz P, Mehlich H (Hrsg) Digitale Transformation von Dienstleistungen im Gesundheitswesen II. Springer Gabler, Wiesbaden, $\mathrm{S} 23-50$

Piecha A, Wegge J, Werth L, Richter PG (2012) Geteilte Führung in Arbeitsgruppen - ein Modell für die Zu- kunft? In: Grote S (Hrsg) Die Zukunft der Führung. Springer, Berlin Heidelberg, S 557-572

Ralfs D (2018) Krankenhaus 4.0, Den Patientenfluss steuern und monitoren. KU Gesundheitsmanagement 6:62-65

Rösler U, Schmidt K, Merda M, Melzer M (2018) Digitalisierung in der Pflege. Wie intelligente Technologien die Arbeit professionell Pflegender verändern. Geschäftsstelle der Initiative Neue Qualität der Arbeit. Bundesanstalt für Arbeitsschutz und Arbeitsmedizin, Berlin

Scheuer E (2017) Wie Medical-Decision-SupportSysteme die Arzt-Patient-Beziehung verändern Digitalisierung von Informationen führt zu einer erhöhten Autonomie des Patienten. In: Pfannstiel MA, Da-Cruz P, Mehlich H (Hrsg) Digitale Transformation von Dienstleistungen im Gesundheitswesen I. Springer Gabler, Wiesbaden, S 311-321

Schirmer U, Woydt S (2016) Mitarbeiterführung, 3. Aufl. Springer, Berlin, Heidelberg

Schneider M, Besser J, Zerth J (2017) Individualisierung durch Digitalisierung am Beispiel der stationären Pflegeversorgung - Organisations- und informationsökonomische Aspekte. In: Pfannstiel MA, DaCruz P, Mehlich H (Hrsg) Digitale Transformation von Dienstleistungen im Gesundheitswesen I. Springer Gabler, Wiesbaden, S 205-226

Teichmann S, Hüning C (2018) Digital LeadershipFührung neu gedacht: Was bleibt, was geht? In: Keuper F, Schomann M, Sikora LI, Wassef R (Hrsg) Disruption und Transformation Management. Springer Gabler, Wiesbaden, S 23-42

Wang D, Waldman DA, Zhang Z (2014) A meta-analysis of shared leadership and team effectiveness. J Appl Psychol 99:181-198

Wegge J, Rosenstiel L (2014) Führung. In: Schuler H, Moser K (Hrsg) Lehrbuch Organisationspsychologie, 5. Aufl. Hans Huber, Bern, S 315-368

Zhu J, Liao Z, Yam KC, Johnson RE (2018) Shared leadership: A state-of-the-art review and future research agenda. J Organ Behav 39:834-852 (https:// doi.org/10.1002 /job.2296. Zugegriffen: 21. November 2018) 
Open Access Dieses Kapitel wird unter der Creative Commons Namensnennung 4.0 International Lizenz (http:// creativecommons.org/licenses/by/4.0/deed.de) veröffentlicht, welche die Nutzung, Vervielfältigung, Bearbeitung, Verbreitung und Wiedergabe in jeglichem Medium und Format erlaubt, sofern Sie den/die ursprünglichen Autor(en) und die Quelle ordnungsgemäß nennen, einen Link zur Creative Commons Lizenz beifügen und angeben, ob Änderungen vorgenommen wurden.

Die in diesem Kapitel enthaltenen Bilder und sonstiges Drittmaterial unterliegen ebenfalls der genannten Creative Commons Lizenz, sofern sich aus der Abbildungslegende nichts anderes ergibt. Sofern das betreffende Material nicht unter der genannten Creative Commons Lizenz steht und die betreffende Handlung nicht nach gesetzlichen Vorschriften erlaubt ist, ist für die oben aufgeführten Weiterverwendungen des Materials die Einwilligung des jeweiligen Rechteinhabers einzuholen.

(c) (1) 